infection at age 5 years. Alopecia areata, psychomotor retardation and ADHD followed, and the diagnosis of MCC deficiency was made at the onset of multiple sclerosis. Urinary organic acids showed an increased excretion of 3-MCG and 3-HIVA, carnitine was decreased, and enzyme measurements in cultured fibroblasts confirmed the 3-MCC deficiency. Response to interferon was poor, but a good response followed treatment with mitoxantrone.

\title{
CHRONIC INFLAMMATORY POLYNEUROPATHY
}

Thirteen children with chronic inflammatory demyelinating polyneuropathy monitored between 1975 and 2005 are reported from Centre hospitalier universitaire SainteJustine, Montreal, Canada. Age at diagnosis varied between 3 and 14 years; 4 were female and 9 male. All presented with symmetrical muscle weakness, mainly in the lower limbs. One had asymmetrical upper limb weakness. Symptoms evolved over 1 week to 1 year, and diagnosis was confirmed after 4 weeks of progression or relapsing course. Sensory symptoms, hypoesthesia and painful paresthesias, occurred in $54 \%(7 / 13)$ of patients at diagnosis. Two patients had diplopia. A respiratory tract viral infection preceded onset in $23 \%(3 / 13)$. Disability varied between minor and severe, 2 to 5 on the Rankin score. Diagnosis was confirmed by delayed nerve conduction, elevated protein $>35 \mathrm{mg} / \mathrm{dL}$ and leukocytes $<10 / \mathrm{mm}^{3}$ in the CSF, and nerve biopsy in 5 patients $(38 \%)$, showing inflammation and segmental demyelination. Evolution was polyphasic in $10(77 \%)$ patients, with 1 to 10 relapses over a follow-up of 6 months to 5 years. Patients treated before 2000 (7/13) received prednisone with good response. Some required repeat courses at relapse and some received azathioprine or cyclophosphamide in addition. After 2000, 3 of 4 patients received intravenous immunoglobulin with an excellent initial response. All required repeat treatments for relapses, including add-on therapies in 2. Two recent patients are refractory, but their disabilities are only minimal to minor. (Rossignol E, D'Anjou G, Lapointe N, Haddad E, Vanasse M. Evolution and treatment of childhood chronic inflammatory polyneuropathy. Pediatr Neurol Feb 2007;36:88-94). (Respond: Dr Vanasse, Service de neurologie, 5 Bloc 4, Centre hospitalier universitaire Ste-Justine, 3175 Cote Ste-Catherine, Montreal, Quebec, Canada H3T 1C5).

COMMENT. Chronic inflammatory demyelinating polyneuropathy (CIDP) is an acquired progressive symmetrical proximal weakness with areflexia, associated with paresthesias or ataxia. Presentation in children is more subacute than chronic, with numerous relapses. Prognosis is usually favorable with complete functional recovery. In the above study, most had relapses requiring repeat courses of prednisone and add-on therapies, and some cases were refractory to treatment.

In a report of 39 patients with CIDP treated with either high-dose, intermittent IV methylprednisolone (IV MP), IV immunoglobulin (IVIg) or oral prednisone or cyclosporine, 81 to $88 \%$ showed improvement in muscle strength, and these treatments were equally effective. Weight gain and cushingoid features were less frequent in patients treated with IVMP (19\% affected) than in those receiving oral prednisone (58\%). IVMP as initial and maintenance therapy is recommended in CIDP patients with weakness or disability (Lopate $G$ et al. Arch Neurol 2005;62:249-254; Ped Neur Briefs March 2005;19:18). 
Mitochondrial neurogastrointestinal encephalomyopathy in an 18-year old female presented with symptoms suggestive of relapsing CIDP, but the gastrointestinal symptoms and lactic acidosis were not compatible (Ginsberg L et al. The Lancet Neurology February 2007;6:192-198).

Noninvasive ventilation used in treatment of respiratory failure in children with neuromuscular disease results in a reduction of symptoms, hospitalizations, and health care costs, without adverse effects on quality of life. Fourteen patients were followed for 6 to 84 months (median $30 \mathrm{mo}$ ). Daytime sleepiness and headache, and sleep quality improved, despite disease progression. (Young HK et al. Neurology 2007;68:198-201).

\section{MOVEMENT DISORDERS}

\section{MOVEMENT DISORDERS INDUCED BY ANTIEPILEPTIC DRUGS}

The prevalence and risk factors for the development of movement disorders, mainly parkinsonism and postural tremor, were analyzed in 201 adults ( $>18$ years, mean 40 years) treated with valproate and newer antiepileptic drugs (AED) at the Epilepsy Clinic, Toronto Western Hospital, Ontario, Canada. Postural tremor was the most common movement disorder, occurring in $45 \%$ of patients. Tremor was mild in $70 \%$ and moderate to severe in $30 \%$. Valproate and carbamazepine were most commonly associated with tremor, followed by phenytoin. Parkinsonism developed in $4.5 \%$, and the odds of having parkinsonism was 5 times higher with valproate than with other AEDs. Five of 9 patients with parkinsonism were concurrently using other drugs or had comorbidities, that could have caused or exacerbated the parkinsonism. Dystonia diagnosed in 4 patients presented with writer's cramp (with carbamazepine, clobazam and topiramate in 1, carbamazepine and valproate in 1), and foot inversion and hand posturing in 1 with a history of cerebral palsy (with topiramate). Tics manifested by excessive blinking developed in 2 patients, one on a combination of phenytoin and clobazam, and the other on valproate. None of the newer AEDs was clearly associated with movement disorders, and these included lamotrigine, vigabatrin, levetiracetam, and oxcarbazepine, but the numbers were small. (Zadikoff C, Munhoz RP, Asante AN et al. Movement disorders in patients taking anticonvulsants. J Neurol Neurosurg Psychiatry February 2007;78:147-151). (Respond: Dr C Zadikoff, Division of Neurology, Toronto Western Hospital, University of Toronto, Toronto, Ont, Canada).

COMMENT. Postural tremor is a common side effect of AEDs in adults and especially with valproate (VPA). In children, VPA also causes tremor and choreiform movements (Lancman ME et al. Arch Neurol 1994;51:702-704). The chorea was dose related; it resolved when VPA was withdrawn or replaced by divalproex sodium sprinkles. Reversible dementia and brain atrophy are reported during VPA therapy in 2 children ages 8 and 10 years. (Papazian O et al. Ann Neurol 1995;38:687-691). 